\title{
A TEORIA INSTITUCIONAL E SUA APLICAÇÃO NA PERSPECTIVA DE SISTEMAS DE INFORMAÇÃO
}

\author{
http://dx.doi.org/10.21527/2237-6453.2021.56.11758
}

Recebido em: 26/11/2020

Aceito em: 11/5/2021

\section{Daiana de Marco, ${ }^{1}$ Carolina Schneider Bender, ${ }^{2}$ Debora Bobsin ${ }^{2}$}

\section{RESUMO}

A Teoria Institucional apresenta importantes contribuições para os estudos organizacionais e para a investigação das influências que moldam as estruturas, esquemas, regras, normas e comportamento dos atores. Nesse sentido, torna-se uma lente de pesquisa interessante para o estudo dos fenômenos inerentes à área de Sistemas de Informações (SI). Esta pesquisa apresenta um panorama da interface entre a Teoria Institucional e a área de Sistemas de Informação, por meio de uma pesquisa bibliométrica realizada na base de dados Web of Science. Somada à análise das características gerais das 128 publicações encontradas, foi utilizada a técnica de acoplamento bibliográfico, buscando a compreensão da rede social temática. De forma a traçar a perspectiva metodológica adotada pelos pesquisadores de SI ao utilizarem a Teoria Institucional, foi realizada uma análise aprofundada das 20 publicações com o maior número de citações. Os resultados revelam que a Teoria Institucional apresenta inúmeras aplicabilidades nos estudos de SI e, também, são apontados caminhos de pesquisa consolidados, bem como sugestões de aplicações da Teoria Institucional que podem ser explorados pelos pesquisadores de SI.

Palavras-chave: teoria institucional; sistemas de informação; bibliométrico; acoplamento bibliográfico.

\section{INSTITUTIONAL THEORY AND ITS APPLICATION IN THE INFORMATION SYSTEMS PERSPECTIVE}

\section{ABSTRACT}

Institutional Theory presents important contributions to organizational studies in relation to the investigation of influences that shape the structures, schemes, rules, norms and actor behavior. Institutional Theory becomes an interesting research lens for the study of phenomena inherent to the Information Systems (IS) area. This study presents an overview of the interface between Institutional Theory and the IS area through a bibliometric research carried out in the Web of Science database. In addition to the analysis of the general characteristics of the 128 publications founded at Web of Science, the bibliographic coupling technique was used, seeking to understand the thematic social network. In order to outline the methodological perspective adopted by IS researchers when using Institutional Theory an analysis of the 20 publications with the highest number of citations was carried out. The results reveal that Institutional Theory has numerous applications in IS studies, point out established research paths and suggest Institutional Theory applications that may be explored by IS researchers.

Keywords: institutional theory; information systems; bibliometric; bibliographic coupling.

\footnotetext{
${ }^{1}$ Autora correspondente. Universidade Federal de Santa Maria (UFSM). Av. Roraima, n. 1000 - Cidade Universitária - Bairro Camobi. Santa Maria/RS, Brasil. CEP 97105-900. http://lattes.cnpq.br/3901996119470340. https://orcid.org/0000-0001-7099-2680. daianademarco2017@gmail.com

${ }^{2}$ Universidade Federal de Santa Maria (UFSM). Santa Maria/RS, Brasil.
} 


\section{INTRODUÇÃO}

A Teoria Institucional apresenta importantes contribuições para os estudos organizacionais no que respeita à investigação das influências que moldam as estruturas, esquemas, regras, normas e comportamento dos atores. Tal entendimento decorre do trabalho de Scott (2008), que propõe as instituições como estruturas sociais que fornecem às organizações ou indivíduos linhas de ação e, ao mesmo tempo, as controlam e restringem. O pressuposto central da Teoria Institucional alicerça-se na tentativa de obtenção de legitimidade pelas organizações e atores, que buscam a aceitação e, consequentemente, a garantia de sua sobrevivência em longo prazo (MEYER; ROWAN, 1977).

Dentro da grande área da Administração, o campo acadêmico de Sistemas de Informação (SI) estuda o impacto da Tecnologia de Informação e Comunicação nas organizações, indivíduos, grupos e sociedade (DINIZ et al., 2016). Na área de SI, é crescente o interesse dos pesquisadores na utilização da Teoria Institucional para examinar fenômenos como inovação, desenvolvimento, instituição, adoção e uso de SI. A Teoria Institucional, dessa forma, apresenta-se como perspectiva alternativa de explicar as organizações, considerando não apenas as ações racionais dos gestores, mas também o contexto institucional no qual eles estão inseridos (MIGNERAT; RIVARD, 2009).

Entre essas contribuições, destaca-se a investigação do desenvolvimento e da adoção de sistemas (GHOLAMI et al., 2013; YOON; GEORGE, 2013; SON; BENBASAT, 2007; CAVALLUZZO; ITTNER, 2004), influência de campos organizacionais e de fatores institucionais (BUTLER, 2011; MARTINSONS, 2008; CHIASSON; DAVIDSON, 2005; DAMSGAARD; LYYTINEN, 1998), segurança (HU; HART; COOKE, 2007), estabelecimento de ERP (BERENTE; YOO, 2012; CURRIE, 2009; JENSEN; KJ/ERGAARD; SVEJVIG, 2009), instituições formais e informais (KSHETRI; PALVIA; DAI, 2011), resposta às pressões institucionais (BHAKOO; CHOI, 2013; RAMILLER; SWANSON, 2003) e ambiente institucional na terceirização em SI (MIRANDA; KIM, 2006).

O presente trabalho buscou apresentar um panorama da interface entre a Teoria Institucional e a área de Sistemas de Informação, por meio de uma pesquisa bibliométrica realizada na base de dados Web of Science (WoS). Foram analisadas as características gerais das publicações e também utilizada a técnica de acoplamento bibliográfico, que ocorre quando dois artigos referenciam, pelo menos, uma publicação em comum (GRÁCIO, 2016). Essa técnica permite estruturar como um conjunto de publicações reflete as abordagens teóricas utilizadas (SASSETTI et al., 2018). Complementarmente, também foi realizada uma análise dos aspectos metodológicos de uma amostra dos 20 artigos mais citados do conjunto de dados. A realização dessas etapas permitiu inferir o estado atual da utilização da Teoria Institucional na área de Sistemas de Informação e a sugestão de caminhos de pesquisa utilizando as abordagens.

\section{TEORIA INSTITUCIONAL}

A partir da publicação do trabalho de Meyer e Rowan (1977), intitulado Institutionalized Organizations: Formal Structure as Myth and Ceremony, a Teoria Institucional ganha destaque na pesquisa organizacional. Os autores desenvolvem a referida teoria com base nas organizações formais da sociedade pós-industrial, abordando que essas instituições refletem mitos, que, por sua vez, são regras institucionalizadas incorporadas pelas organizações, na busca por legitimidade, recursos, estabilidade e melhores perspectivas de sobrevivência (MAYER; ROWAN, 1977). 
Para os autores, as estruturas formais são compreendidas como sistemas de atividades coordenadas e controladas, que irão propiciar o surgimento de organizações formais em ambientes altamente institucionalizados. Esses ambientes, por sua vez, forçam as empresas a incorporarem práticas e procedimentos institucionalizados pela sociedade. Assim, as organizações que adotam essas práticas e procedimentos garantem sua legitimidade em determinado campo institucional, e, consequentemente, a sobrevivência em longo prazo, independentemente de sua eficácia organizacional. As próprias práticas e procedimentos advêm de um processo de institucionalização, baseado em isomorfismos miméticos, normativos e coercitivos (MAYER; ROWAN, 1977).

O isomorfismo, dessa forma, é considerado um mecanismo de mudança institucional. O isomorfismo coercitivo deriva de influências políticas e problemas de legitimidade, sendo provocado por pressões formais e informais de outras organizações e pelas expectativas culturais da sociedade. $\mathrm{O}$ isomorfismo mimético deriva de respostas padronizadas à incerteza, nas quais as organizações tendem a tomar como modelo no campo outras organizações já legitimadas. $\mathrm{O}$ isomorfismo normativo, por sua vez, está associado à profissionalização e aos grupos de indivíduos intercambiáveis entre as organizações. Esses processos garantem a homogeneização das instituições, e, consequentemente, o sucesso e a sobrevivência das organizações (MAYER; ROWAN, 1977).

As influências institucionais operam em três camadas, ou seja, campo social, campo organizacional e organização. Nesta dinâmica, as instituições estruturam os campos organizacionais, criando o ambiente institucional que irá fornecer as estruturas nas quais as organizações operam (BUTLER, 2011). O campo organizacional pode ser entendido como as organizações que produzem serviços ou produtos similares ou atuam em conjunto em uma área como, exemplificam DiMaggio e Powell (2005, p. 76): "fornecedores-chave, consumidores de recursos e produtos, agência regulatórias e outras organizações".

Os processos dentro dos campos organizacionais moldam a complexidade institucional experimentada pelas organizações (SCOTT, 2008), que irão senti-la de maneira e em graus diferentes, dependendo de sua posição no campo e sua estrutura, assim como irão diferir em termos de respostas, que têm implicações tanto para o campo quanto para a sobrevivência organizacional (GREENWOOD et al., 2011). Segundo os autores, "as organizações enfrentam a complexidade institucional sempre que confrontam prescrições incompativeis de múltiplas lógicas institucionais", destacando-se que as lógicas institucionais "fornecem um meio de compreender o mundo social e, portanto, de agir com confiança dentro dele" (GREENWOOD et al., 2011, p. 318) e, assim, obter sucesso dentro do campo organizacional.

A entrada de novas organizações no campo provoca mudanças sociais, proporcionando às organizações do campo a instalação de uma nova lógica institucional ou a priorização das existentes. Sendo assim, essa é a principal fonte da renovação da complexidade institucional no longo prazo, que, segundo Greendwood et al. (2011, p. 319), "se desdobra, se desfaz e se reforma, criando diferentes circunstâncias às quais as organizações devem responder".

No que diz respeito à mudança institucional endógena, cabe salientar o papel dos empreendedores institucionais, na condição de agentes da mudança. A concepção de empreendedorismo institucional foi introduzida na Teoria Institucional como uma forma de compreender como acontecem as mudanças divergentes (DIMAGGIO, 1988). Essas mudanças são aquelas que rom- 
pem com o modelo institucionalizado da organização, e os empreendedores institucionais contribuem para a transformação ou criação de novas instituições (BATTILANA; LECA; BOXENBAUM, 2009). São características do campo, como solavancos, crises, agitação social, interrupção tecnológica, mudanças regulatórias, entre outras, que somadas à posição social do empreendedor institucional, relacionada com o status da organização, autoridade, capital social, posição na sociedade, características individuais e fatores demográficos e psicológicos, que irão determinar a capacidade de mudança desse empreendedor e a produção de efeitos no campo.

Por fim, entende-se que a Teoria Institucional ainda deve percorrer um caminho rumo a sua institucionalização, pois não contém um conjunto de variáveis e nem uma metodologia de pesquisa padrão (TOLBERT; ZUCKER, 1999). Assim, reforçam os referidos autores a importância de estudos que contemplem a temática da Teoria Institucional, para que cada vez mais se aprimore o seu desenvolvimento acadêmico, corroborando a presente pesquisa. A Teoria Institucional tem como convicção que os arranjos institucionais e os processos sociais são importantes na formulação social, havendo ainda um grande número de questões a serem exploradas na literatura que podem ampliar os argumentos institucionais (WOOTEN; HOFFMAN, 2008).

\section{SISTEMAS DE INFORMAÇÃO}

A origem dos estudos de SI dá-se em 1960, a partir das áreas de Ciência da Computação, operações, contabilidade e estudos organizacionais (BRYANT et al., 2013; ZHANG, 2015). Atualmente, abarca também áreas como Computação e Engenharia, porém a maior parte ainda encontra-se vinculado à ciência social, dentro das escolas de negócio (DANTAS; CALLADO, 2018).

A área de Sistemas de Informação (SI), dentro do campo de estudos da Administração, advém da tradução dos termos Management Information Systems (MIS) e Information Systems (IS) (DINIZ et al., 2016), tangenciando assuntos como tecnologia, desenvolvimento, uso e gerenciamento da TI, baseando-se, principalmente, no paradigma funcionalista (RODRIGUES FILHO; LUDMER, 2005).

Com relação às bases teóricas e metodológicas, o campo de SI é alicerçado em bases teóricas e metodológicas de distintas ciências, com pesquisadores de múltiplas origens, como Física, Química, Matemática, Psicologia, Administração, Ciência da Computação, entre outras (DANTAS; CALLADO, 2018). Relativamente à área de Administração, o foco de atuação dos pesquisadores em SI está na análise organizacional, incluindo fundações filosóficas da informação e comunicação (RODRIGUES FILHO; LUDMER, 2005).

Ainda, uma crítica à abordagem de SI trazida por Rodrigues Filho e Ludmer (2005, p. 155) é que embora "seja reconhecido muito mais como um fenômeno social do que um fenômeno puramente técnico, boa parte da pesquisa em SI é bastante limitada pelas suposições de que SI é um fenômeno técnico".

\section{MÉTODO DE PESQUISA}

Esta pesquisa apresenta um panorama da interface entre a Teoria Institucional e a área de Sistemas de Informação, por meio de uma busca realizada na base de dados Web of Science (WoS). Assim, no que se refere à orientação metodológica adotada, caracteriza-se como biblio- 
métrica. Tal procedimento destina-se a quantificar, identificar, analisar e descrever uma série de padrões que permitem analisar o comportamento científico ou técnico de um determinado campo do conhecimento por meio do estudo quantitativo de publicações (ARAÚJO, 2006). Dessa forma, permite examinar a evolução de domínios de pesquisa e autores, com base nas estruturas sociais, intelectuais e conceituais dos tópicos de análise (DONTHU; KUMAR; PATTNAIK, 2020).

A bibliometria possui três leis clássicas: Lei de Lotka, que estabelece a lei dos quadrados inversos, podendo assim investigar a produtividade dos autores em várias disciplinas, proporcionando a identificação de centros de pesquisas mais desenvolvidos e a consolidação de uma área científica (ARAÚJO, 2006); a Lei de Bradford tem o objetivo de investigar a distribuição dos artigos nos periódicos, auxiliando na determinação do periódico mais relevante sobre determinado tema e a Lei de Zipf, "que descreve a relação entre palavras num determinado texto suficientemente grande e a ordem de série destas palavras" (ARAÚJO, 2006, p. 16), ou seja, é a contagem de palavras e a sua relevância para uma área de pesquisa.

A primeira etapa da análise bibliométrica é a definição do escopo do estudo, sendo a escolha da base, bem como das palavras-chave, aspectos que impactam significativamente sobre a validade da pesquisa (SINGLETON et al., 1999). Sendo assim, a escolha da WoS se justifica pela sua reputação diante da indexação dos periódicos mais citados em cada área. Além disso, a base permite a elaboração de rankings por diversos parâmetros que atendiam às necessidades deste estudo.

Com relação às palavras-chave, optou-se pela utilização dos termos "institucional theory" e "information* system*". A escolha pela técnica de truncagem, idealizada por meio do símbolo "*" após as palavras descritas, possibilita a utilização da raiz da palavra na recuperação das diversas possibilidades de sua expansão (LOPES, 2002), ampliando, assim, a busca dos termos. Também foi utilizado o operador de pesquisa "AND", que tem como função restringir a recuperação de materiais que contenham ambos os termos separados pelo operador. A busca foi realizada no rótulo de campo de pesquisa avançada, utilizando como marcador de seleção "Tópico", que inclui Título, Resumo, Palavras-chave e Indexação como Termos Taxonômicos, Sistemáticos e Descritores (WEB OF SCIENCE, 2020). Não foram delimitadas áreas, tampouco tipo de documentos e ano. Desta forma, o período compreendeu entre 1945 e 2020.

A busca foi realizada em janeiro de 2020 da seguinte forma: TS = "institutional theory" AND "information* system*”, retornando 128 publicações. Essa etapa caracterizou a seleção inicial do presente estudo, fornecendo dados para a análise bibliométrica. Esta foi dividida em três etapas: i) análise das características gerais; ii) análise da rede social temática e iii) análise da perspectiva metodológica. A Figura 1 resume a estratégia de pesquisa adotada.

Com relação à primeira, por meio das ferramentas disponíveis na WoS foram gerados relatórios e gráficos que permitiram a identificação das características gerais das produções científicas. Foram realizadas análises relativas ao ano das publicações, países, autores, instituições e áreas temáticas.

Buscando a compreensão da estrutura teórica das publicações, segunda etapa, foi realizada uma análise de acoplamento bibliográfico no software VOSviewer. Essa técnica fornece um grande conjunto de informações concentradas em um único gráfico, no qual a distância entre os itens pode ser interpretada como uma indicação da relação entre os termos, tornando-se mais forte à medida em que se aproximam formando clusters (VAN ECK et al., 2010). 


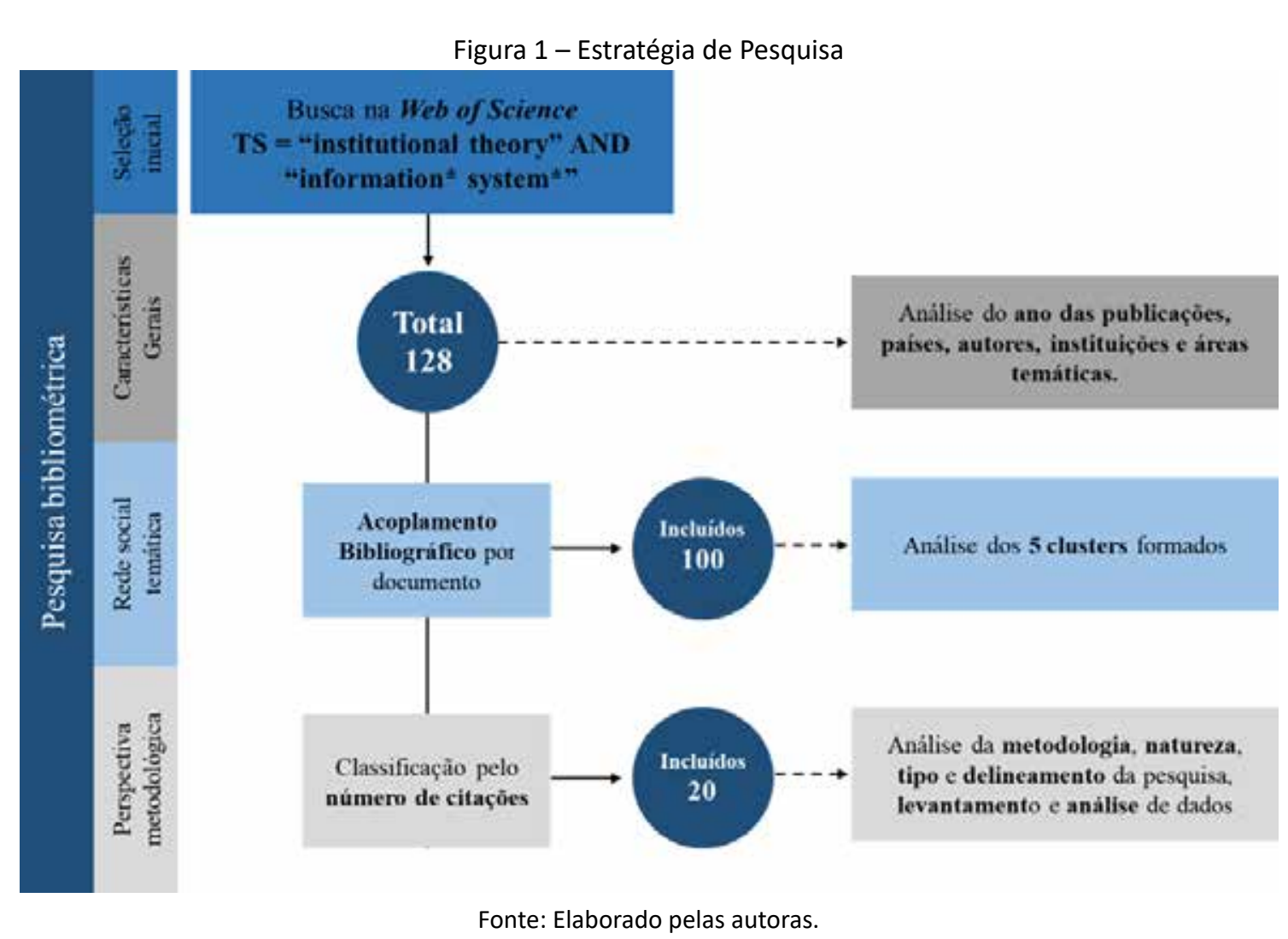

Itens que pertencem a um mesmo agrupamento estão fortemente vinculados, como um grupo que compartilha das mesmas referências, sinalizando que um cluster representa um fluxo de pesquisa ou um tópico específico em uma base de similaridade. A técnica de acoplamento bibliográfico foi instituída por meio dos documentos, considerando o mínimo de uma citação. Formaram-se 5 clusters (vermelho, verde, roxo, azul e amarelo), caracterizados conforme a densidade dos conglomerados, e o perfil temático de cada um foi traçado a partir da descrição dos 5 artigos mais citados dentro do cluster.

Visando a complementar as análises das características gerais e da rede social temática anteriormente detalhadas, sentiu-se a necessidade de traçar a perspectiva metodológica adotada pelos pesquisadores ao investigarem temáticas atinentes à área de SI utilizando a Teoria Institucional, o que constitui a terceira etapa do estudo bibliométrico, conforme exposto na Figura 1. Dessa forma, as 128 publicações foram submetidas a uma nova triagem, tendo por critério de classificação o número de citações e como objetivo a análise em profundidade dos 20 mais citados. Foram selecionados, em um primeiro momento, 25 artigos, os quais foram examinados e classificados de forma independente por duas pesquisadoras. A margem de 5 artigos deu-se para o caso de alguma publicação não se encaixar à temática proposta e necessitar ser excluída.

Concluída a classificação individual, os resultados foram confrontados e, não havendo divergência entre as pesquisadoras, decidiu-se pela exclusão das últimas 5 publicações entre as 25 selecionadas. Assim, os 20 artigos mais citados foram analisados a partir de sua metodologia, natureza, tipo e delineamento da pesquisa, forma de levantamento e de análise dos dados, e, ainda, considerando-se a aplicação da área de Sistemas de Informação e da Teoria Institucional. Entende-se que a idealização das três etapas propostas foi essencial para a obtenção do panorama do campo. 


\section{RESULTADOS}

\section{Características Gerais}

A primeira etapa da coleta foi realizada no sistema de busca por tópicos da Principal Coleção da WOS, seguindo-se os passos explanados na seção anterior. Visando a verificar o comportamento da produção científica ao longo do tempo, demonstra-se a distribuição quantitativa dessas publicações em cada período, conforme Tabela 1. Foram incluídos na tabela apenas os anos em que o número de publicações era maior ou igual a 1.

Tabela 1 - Número de publicações por ano

\begin{tabular}{cccccccc}
\hline Ano & $\begin{array}{c}\text { No de } \\
\text { Publicações }\end{array}$ & Ano & $\begin{array}{c}\text { No de } \\
\text { Publicações }\end{array}$ & Ano & $\begin{array}{c}\text { No de } \\
\text { Publicações }\end{array}$ & Ano & $\begin{array}{c}\text { No de } \\
\text { Publicações }\end{array}$ \\
\hline $\mathbf{1 9 9 8}$ & 1 & 2006 & 3 & 2011 & 4 & 2016 & 13 \\
\hline $\mathbf{2 0 0 2}$ & 1 & 2007 & 3 & 2012 & 7 & 2017 & 11 \\
\hline $\mathbf{2 0 0 3}$ & 2 & 2008 & 8 & 2013 & 11 & 2018 & 10 \\
\hline $\mathbf{2 0 0 4}$ & 4 & 2009 & 13 & 2014 & 9 & 2019 & 13 \\
\hline $\mathbf{2 0 0 5}$ & 1 & 2010 & 3 & 2015 & 9 & $2020^{*}$ & 2 \\
\hline
\end{tabular}

*Considerando apenas o mês de janeiro/2020

Fonte: Elaborado pelas autoras com base na Web Of Science.

Os resultados evidenciam que as primeiras publicações indexadas na base de dados da WoS que utilizam a Teoria Institucional em pesquisa na área de Sistemas de Informação são registradas a partir de 1998. Nos anos de 2008 e 2009 apresenta-se um crescimento expressivo no número de publicações com relação aos anos anteriores, respectivamente 8 e 13 . E, após uma pequena queda entre 2010 e 2012, a partir de 2013 sempre foram registradas, no mínimo, 9 publicações. Por fim, no primeiro mês de 2020, referência da busca na base de dados, já foram indexadas 2 publicações.

Constituiu-se também como objeto de análise os países que predominam nas publicações, o que permite a identificação de grandes centros de pesquisa. Há uma larga predominância de estudos nos Estados Unidos (43 publicações), o que confirma uma tendência já apresentada em pesquisas anteriores envolvendo a Teoria Institucional (MOTKE; DA SILVA RAVANELLO; RODRIGUES, 2016; DE MELO PEREIRA, 2012). Corroboram esse dado as evidências de que a própria pesquisa em SI apresenta-se estabelecida a partir das escolas de negócio norte-americanas (RODRIGUES FILHO; LUDMER, 2005).

Ainda relativo aos países, destaca-se a Inglaterra, com 23 publicações. Individualmente os demais países europeus não apresentam grande frequência, porém, se somados, Inglaterra (23), Noruega (7), Portugal (6), França (5), Irlanda (5), Dinamarca (4), Finlândia (3), Alemanha (3), Escócia (3) e Espanha (3), totalizam 62 publicações para o continente europeu, configurando-se como uma importante bolsa de pesquisa de Sistemas de Informações utilizando a Teoria Institucional. Se somados os números dos Estados Unidos (43) aos estudos do Canadá (10), evidencia-se a América do Norte (53) como o segundo continente em termos de representatividade.

Com relação aos autores com maior número de publicações, a Tabela 2 apresenta o referido levantamento, limitando-se aos 10 primeiros. Os autores que mais publicaram sobre $o$ tema foram Hossein Ahmadi e Amizan Omar, contabilizando 4 artigos por autor, seguidos de 
7 autores que publicaram 3 artigos. Os 128 trabalhos analisados contemplam um total de 248 autores envolvidos, entre os quais 219 publicaram apenas 1 artigo. Pela variedade de autores infere-se que o tema de estudo ainda se encontra em construção.

Tabela 2 - Principais autores

\begin{tabular}{|c|c|c|}
\hline Posição & Autores & Registros \\
\hline $1 \underline{a}$ & AHMADI H & 4 \\
\hline $2 \mathrm{a}$ & OMAR A & 4 \\
\hline 3a & ALSHARARI NM & 3 \\
\hline $4 a$ & BUTLER T & 3 \\
\hline 5 & HAYES N & 3 \\
\hline 6a & NILASHI M & 3 \\
\hline $7 \underline{a}$ & OLIVEIRA T & 3 \\
\hline $8 \mathrm{a}$ & WEERAKKODY V & 3 \\
\hline 9a & ZHANG C & 3 \\
\hline $10 \mathrm{a}$ & BARTON KA & 2 \\
\hline Total & & 31 \\
\hline
\end{tabular}

Fonte: Adaptado de Web of Science (2020).

As principais instituições associadas às publicações também constituem um dado relevante para a bibliometria, pois demonstram locais profícuos no que tange à temática da pesquisa. Nesse viés, a Tabela 3 apresenta um ranking das dez organizações que mais contribuíram para a compreensão do tema pesquisado.

Tabela 3 - Principais instituições associadas às publicações

\begin{tabular}{cll}
\hline Posição & \multicolumn{1}{c}{ Organizações } & Registros \\
\hline \multirow{2}{*}{$\mathbf{a}$} & BRUNEL UNIVERSITY & 5 \\
\cline { 2 - 3 } & HEC MONTREAL & 5 \\
\cline { 2 - 3 } & UNIVERSITY OF MONTREAL & 5 \\
\cline { 2 - 3 } & UNIVERSITY OF NORTH CAROLINA & 5 \\
\cline { 2 - 3 } & UNIVERSITY SYSTEM OF GEORGIA & 5 \\
\hline $\mathbf{6} \mathbf{a}$ & UNIVERSITY OF WARWICK & 4 \\
\hline $\mathbf{7 0}$ & ISLAMIC AZAD UNIVERSITY & 3 \\
\cline { 2 - 3 } & LANCASTER UNIVERSITY & 3 \\
\cline { 2 - 3 } & NATIONAL TAIWAN UNIVERSITY & 3 \\
\cline { 2 - 3 } & STATE UNIVERSITY SYSTEM OF FLORIDA & $\mathbf{4 1}$ \\
\hline Total & &
\end{tabular}

Fonte: Adaptado de Web of Science (2020).

Verifica-se que há cinco instituições com o maior número de publicações, sendo elas Brunel University, localizada na Inglaterra, HEC Montreal e University of Montreal, ambas localizadas no Canadá, e University of North Carolina e University System of Georgia, localizadas nos Estados Unidos. Tais dados corroboram as informações apresentadas no que respeita aos principais países e continentes das publicações, nos quais evidenciou-se dois grandes bolsões de pesquisas na América do Norte e na Europa. 
Com a finalidade de elencar como as principais áreas temáticas de pesquisa estão relacionadas ao tema proposto para o presente estudo, foram assinaladas, conforme número de registros, as dez áreas de maior destaque (Tabela 4). Assim, destaca-se como primeiro lugar a área Computer Science, com 71 publicações, seguida das áreas de Business Economics (65) e Information Science Library Science (65).

Tabela 4 - Principais áreas temáticas de pesquisa

\begin{tabular}{clc}
\hline Posição & \multicolumn{1}{c}{ Organizações } & Registros \\
\hline $\mathbf{1 a}$ & COMPUTER SCIENCE & 71 \\
\hline $\mathbf{2} \mathbf{a}$ & BUSINESS ECONOMICS & 65 \\
& INFORMATION SCIENCE LIBRARY SCIENCE & 65 \\
\hline $\mathbf{4} \mathbf{a}$ & ENGINEERING & 6 \\
\cline { 2 - 3 } & SOCIAL SCIENCES OTHER TOPICS & 6 \\
\hline $\mathbf{6 a}$ & PUBLIC ADMINISTRATION & 5 \\
\hline $\mathbf{7} \underline{\mathbf{a}}$ & OPERATIONS RESEARCH MANAGEMENT SCIENCE & 4 \\
\cline { 2 - 3 } & PSYCHOLOGY & 4 \\
\hline $\mathbf{9 a}$ & HEALTH CARE SCIENCES SERVICES & 3 \\
\cline { 2 - 3 } & MEDICAL INFORMATICS & 3 \\
\hline
\end{tabular}

Fonte: Adaptado de Web of Science (2020).

Tais dados seguem a lógica da própria origem dos estudos acerca de Sistemas de Informação, a partir dos estudos da Ciência da Computação (BRYANT et al., 2013; ZHANG, 2015), à qual se alinham "Computer Science" e "Information Science Library Science", vinculados também às escolas de negócios (DANTAS; CALLADO, 2018), que abarcam, por exemplo, tópicos como "Business Economics".

Além disso, a Tabela 4 também permite concluir que as demais áreas temáticas apontadas constituem-se como campos que permitem uma interface entre SI e Teoria Institucional, uma vez que já existem publicações, porém ainda carecem de uma ampliação, tendo em vista o seu baixo número de registros. Nesse sentido, sugere-se que pesquisadores busquem a relação da área de Sistemas de Informação com a Teoria Institucional, como um caminho de pesquisa que pode gerar conhecimentos adicionais.

\section{Rede Social Temática}

Buscando-se compreender o fluxo da pesquisa envolvendo dentro da área de SI a Teoria Institucional, foi realizado o procedimento de acoplamento bibliográfico, que evidencia agrupamentos e a evolução dos estudos (SASSETI et al., 2008). O agrupamento dos documentos a partir das citações em comum evidencia a existência de semelhança cognitiva, ou seja, que os clusters formados representam trabalhos dentro de uma mesma especialidade de pesquisa (JARNEVING, 2007).

O acoplamento bibliográfico resultou em cem documentos com no mínimo uma citação, criando 5 clusters, conforme apresentado na Figura 2. Foram analisados e descritos os 5 artigos mais citados de cada cluster, visando a traçar o perfil das pesquisas e temáticas atinentes. 


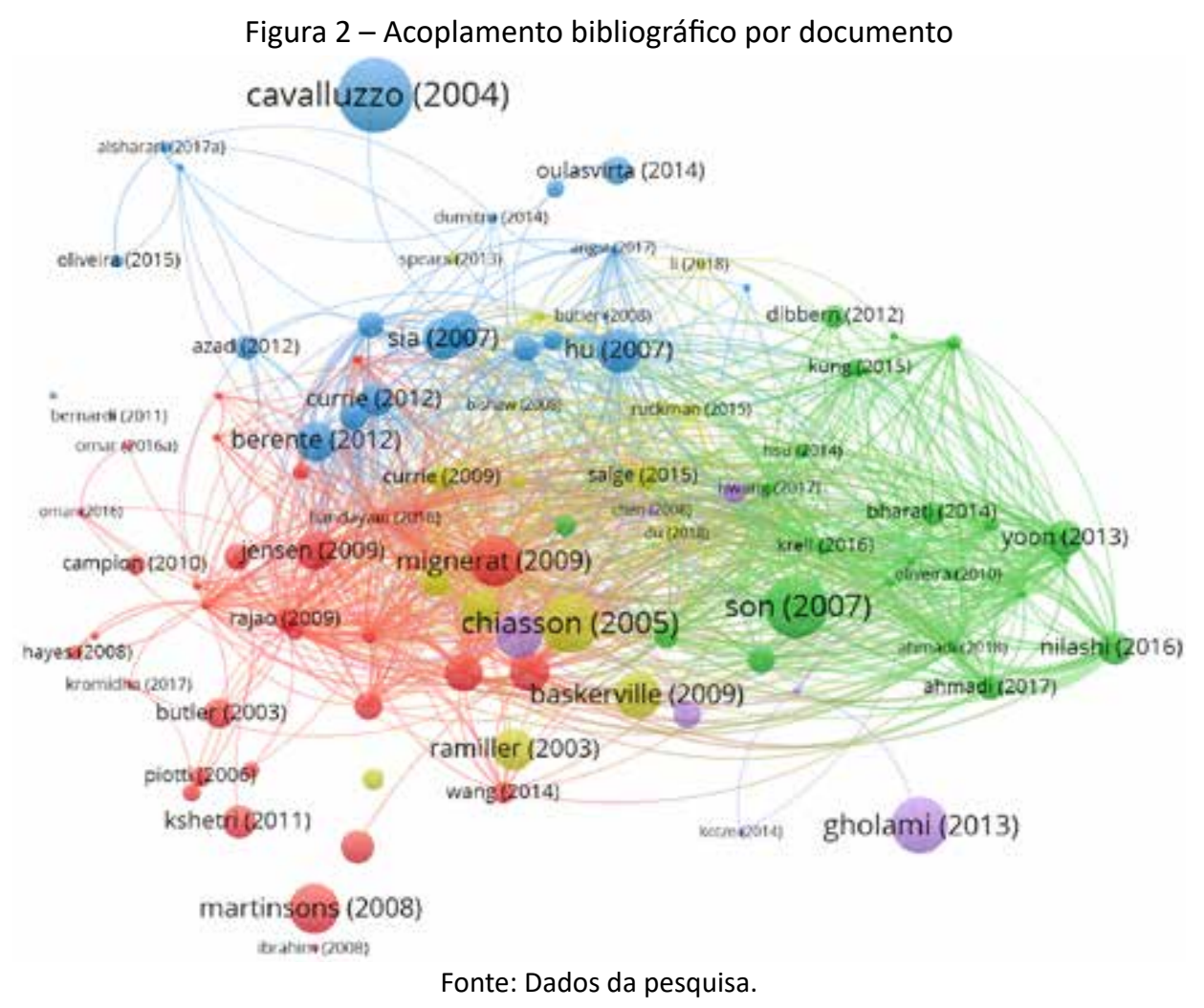

O cluster vermelho é composto por 33 publicações que investigam os efeitos institucionais, abordando fenômenos como inovação, adoção, instituição e assimilação de SI. São analisadas as regras formais e convenções informais que reduzem a incerteza no e-commerce, fornecendo uma estrutura para interações políticas, sociais e econômicas (MARTINSONS, 2008). Ainda, são investigadas as pressões institucionais, tanto na adoção de SI nos setores público e privado e em âmbito individual e organizacional (BHAKOO; CHOI, 2013; BUTLER, 2003; MIGNERAT; RIVARD, 2009); quanto nos desafios de pesquisa pertinentes à área de sistemas de informação (WEERAKKODY; DWIVEDI; IRANI, 2009).

O segundo cluster analisado, de cor verde, é composto por 22 artigos que se referem à intenção de adoção de SI por organizações públicas e privadas. Os cinco estudos mais citados envolvem a influência da eficiência e legitimidade em mercados eletrônicos business-to-business (B2B) (SON; BENBASAT, 2007); a lentidão e barreiras na adoção de mundos virtuais (YOON; GEORGE, 2013); sistemas de informações hospitalares (NILASHI et al., 2016; AHMADI et al., 2016); e sistemas de informação de governo a governo (G2G) na administração pública (ZHENG et al., 2013). Interessante destacar, no que se refere à Teoria Institucional, que os estudos convergem ao investigarem pressões institucionais - coercitivas, normativas e miméticas - como construtos que impulsionam ou inibem a intenção de adoção de SI.

O cluster azul também compreende 22 artigos, os quais versam sobre as lógicas institucionais na introdução e uso de SI organizacionais. As investigações mais citadas tratam de sistemas de avaliação de desempenho em órgãos governamentais (CAVALLUZZO; ITTNER, 2004); influências internas e externas na segurança em SI (HU; HART; COOKE, 2007); interação entre fornecedores de softwares com as estruturas institucionais organizacionais (SIA; SOH, 2007); 
tomadas de decisão na terceirização de SI (MIRANDA; KIM, 2006) e desafios da efetivação de SI na Nasa (BERENTE; YOO, 2012).

O cluster amarelo, formado por 16 artigos, foca nos processos de institucionalização e na interface de pesquisa entre SI e Teoria Institucional. Destaca-se a análise da dinâmica de institucionalização de SI (NIELSEN; MATHIASSEN; NEWELL, 2014); das respostas de executivos à inovação em SI e seu consequente impacto na difusão e adoção (RAMILLER; SWANSON, 2003); da influência da indústria e da carência de pesquisas empíricas nesse setor na área de SI (CHIASSON; DAVIDSON, 2005); da utilização da Teoria Institucional na área de Sistemas de Informação a partir de uma revisão das principais teorias (CURRIE, 2009) e da utilização da Teoria Institucional e de Teorias da Inovação na análise de modas de pesquisa em SI (BASKERVILLE; MYERS, 2009).

Por fim, o cluster roxo é formado por 7 artigos, que se referem à adoção de SI verde. As publicações propõem modelos conceituais para identificação de fatores externos e internos e uma custos indiretos associados aos investimentos em SI verde (JONGSAGUAN; GHONEIM, 2017); indicam a inclusão de fatores financeiros como determinantes para adoção de SI verde (KOTZE; VAN BELLE; MCGIBBON, 2014); discorrem sobre um modelo que mostre a percepção dos gerentes sobre os antecedentes e as consequências da adoção do SI verde (GHOLAMI et al., 2013); investigam o gerenciamento eletrônico de recursos humanos e SI estratégico em corporações multinacionais (HEIKKILÄ, 2013) e analisam a influência da autoridade na adoção de SI nas organizações governamentais (STANDING; SIMS; LOVE, 2009).

\section{Perspectiva Metodológica}

Nesta seção apresenta-se a análise de uma amostra dos 20 artigos com o maior número de citações entre o conjunto total, buscando complementar as análises anteriores. Tal exploração justifica-se pelo entendimento de que a aplicação da Teoria Institucional ainda não tem um padrão de metodologia e de variáveis que podem ser aplicadas à prática e à pesquisa acadêmica. Após a leitura, foram identificados a natureza, o tipo e o delineamento da pesquisa, assim como a forma de levantamento e de análise dos dados. Ainda foram consideradas a tecnologia investigada e como a Teoria Institucional foi aplicada nos estudos.

Os dados quanto à natureza e ao tipo de pesquisa são expostos na Tabela 5.

Tabela 5 - Natureza e tipo de pesquisa

\begin{tabular}{lclc}
\hline Natureza & No artigos & Tipo & № artigos \\
\hline Qualitativa & 12 & Descritiva & 7 \\
\cline { 3 - 4 } & & Exploratória & 2 \\
\cline { 3 - 4 } & & Explicativa & 1 \\
\cline { 2 - 4 } & & Interpretativista & 2 \\
\hline Quantitativa & 6 & Descritiva & 2 \\
\hline Qualitativa-Quantitativa & 2 & Exploratória & \multicolumn{2}{c}{$\mathbf{2 0}$} \\
\hline Total & & \multicolumn{20}{c}{} \\
\hline
\end{tabular}

Fonte: Elaborado pelos autores.

Com relação à natureza da pesquisa, $60 \%$ (12) artigos são qualitativos, $30 \%$ (6) são quantitativos e $10 \%$ (2) são mistos. No que respeita ao tipo de pesquisa, os estudos qualitativos apre- 
sentam-se mais diversos, uma vez que 35\% (7) destes são descritivos, $10 \%$ (2) são exploratórios, $5 \%$ (1) explicativo e $10 \%$ (2) são interpretativistas.

Quanto a esses últimos, cabe destacar que a origem dos estudos na área de SI é positivista-funcionalista, porém a utilização de múltiplos métodos, teorias e enfoques filosóficos abre espaço para que novos paradigmas adentrem na área, tornando possível refletir a natureza multifacetada da realidade social e organizacional (RODRIGUES FILHO; LUDMER, 2005). Em âmbito nacional, Dantas e Callado (2018) buscaram compreender o processo histórico de introdução e legitimação da área de SI no Brasil, e observam que a construção da identidade social do campo no Brasil ao longo dos anos tem permitido a fuga de estereótipos metodológicos historicamente positivistas.

No que diz respeito ao delineamento e ao levantamento de dados, $6(30 \%)$ estudos do conjunto de 20 analisados foram realizados por meio de estudos de caso, com os dados sendo levantados mediante análise documental, entrevistas, observação, grupo focal e questionário. Também 6 artigos (30\%), utilizaram a técnica de pesquisa survey. Destaca-se que destes, 2 estudos utilizaram outras técnicas em conjunto com a pesquisa survey, como entrevistas de campo e dados secundários. Os estudos bibliométricos, que permitem a identificação do estado atual de um campo de pesquisa, correspondem a $20 \%$ (4). Ainda, $10 \%$ (2) caracterizam-se como revisões da literatura, 5\% (1) como abordagem histórica e 5\% (1) como teoria fundamentada.

Uma vez que utilizam o mesmo método da presente pesquisa, destacam-se alguns pontos importantes das 4 publicações (BASKERVILLE; MYERS, 2009; CHIASSON; DAVIDSON, 2005; MIGNERAT; RIVARD, 2009; WEERAKKODY; DWIVEDI; IRANI, 2009), que utilizaram o levantamento de dados a partir da bibliometria. O estudo de Mignerat e Rivard (2009) objetivou realizar um balanço de como a Teoria Institucional estava sendo utilizada na pesquisa de Sistemas de Informações, utilizando como base 22 periódicos da área, analisando um total de 53 documentos. Os autores encontraram três temas principais de pesquisas: efeitos institucionais, processo de institucionalização e interação entre TI e instituições. Os autores concluíram que a pesquisa institucional em SI é frutífera, porém ainda há muito a ser explorado para abordar completamente a riqueza da Teoria Institucional.

Na mesma linha, Weerakkody, Dwivedi e Irani (2009) propuseram estudo bibliométrico a fim de verificar o atual estado da utilização da Teoria Institucional na área de $\mathrm{SI}$, a partir da análise de 511 artigos publicados em 210 revistas, no período de 1978 a 2008. Os resultados corroboram a presente pesquisa, apontando que tal interface se desenvolve sob o paradigma positivista, a partir da pesquisa empírica e quantitativa.

De forma diferente da presente proposta e dos artigos de Mignerat e Rivard (2009) e Weerakkody, Dwivedi e Irani (2009), o estudo de Baskerville e Myers (2009) utilizou a Teoria Institucional em uma pesquisa bibliométrica que visou a identificar modas de pesquisa na área de Sistemas de Informação. Já Chiasson e Davidson (2005), partindo dos níveis de análise propostos pela Teoria Institucional, buscaram compreender como o contexto da indústria influencia nos tópicos de pesquisa em SI. Os autores analisaram as publicações de pesquisa em SI dos periódicos MIS Quartertly e Information System Research, entre 1997 e 2004, não encontrando evidências de utilização da indústria na construção e teste de teorias na referida área.

Por fim, ainda com relação aos procedimentos metodológicos, destaca-se que as análises dos dados dos estudos apreciados foram realizadas por meio de modelagem de equações estru- 
turais, procedimentos de codificação, procedimentos bibliométricos, categorização, análise de conteúdo, análise fatorial exploratória, análise fatorial, estatística descritiva e correlação bivariada.

No que respeita à forma como a área de SI foi abordada na amostra, destaca-se que, além dos estudos que a investigam de uma forma geral, como os bibliométricos anteriormente descritos e as revisões da literatura, são abordados: segurança, desempenho, desalinhamento, fator humano, instauração e terceirização de SI, Sistemas ERPs, TI Verde, e-commerce, mundos virtuais, tecnologia $3 \mathrm{G}$ e intercâmbio de dados eletrônicos. Os dados apresentados expõem a diversidade de temáticas de investigação em SI que permitem a utilização da Teoria Institucional.

Por fim, destaca-se que os elementos da Teoria Institucional como legitimidade, pressões isomórficas, influências regulativas, normativas e culturais-cognitivas, mitos racionalizados e lógicas institucionais, foram utilizados como construtos em modelos teóricos envolvendo SI. Além disso, também foram investigados processos de institucionalização, a influência dos contextos institucionais nas decisões, intenção de adoção de SI e análise de campos organizacionais.

\section{CONSIDERAÇÕES FINAIS}

A Teoria Institucional constitui-se como uma perspectiva valiosa para estudar as mudanças organizacionais baseadas em SI (ROBEY; BOURDREAU, 1999). A análise realizada nesta pesquisa aponta para inúmeros fenômenos que relacionam Teoria Institucional e Sistemas de Informação que foram estudados ou podem ser importantes agendas de pesquisa futuras. Com a finalidade de contribuir para a compreensão dessa perspectiva, este artigo atingiu o objetivo de traçar um panorama da interface entre a Teoria Institucional e a área de Sistemas de Informação.

Para alcançar o referido objetivo desenvolveu-se uma pesquisa bibliométrica utilizando a base de dados Web of Science, com a inserção dos termos "institutional theory" e "information* system*". A busca retornou 128 publicações, a partir das quais foram realizadas análises das características gerais das publicações, envolvendo números por ano, países, autores, instituições e áreas temáticas, e da rede social temática, por meio do procedimento de acoplamento bibliográfico. Diante da necessidade de um aprofundamento da compreensão da perspectiva metodológica, selecionou-se uma amostra dos 20 artigos com maior número de citações, analisando-os quanto à natureza, tipo e delineamento da pesquisa e levantamento e análise de dados.

Os resultados da primeira etapa expõem um crescimento expressivo do número de publicações nos anos de 2008 e 2009 e, a partir de 2013, um mínimo de 9 publicações por ano, destacando o crescimento do interesse de pesquisadores em adotar a perspectiva da Teoria Institucional nos estudos envolvendo a área de Sistemas de Informação, principalmente se considerado que até 1998 não houve registro na WoS de nenhuma publicação relacionada às duas temáticas.

Também foi confirmada a tendência de predominância dos Estados Unidos, que apresentam 43 do total de publicações, bem como a divisão da maioria das publicações entre a Europa (62) e América do Norte (53), constituindo-se dois grandes blocos de pesquisa. Tais informações foram comparadas com o ranking das 10 instituições associadas às publicações, no qual destacam-se Brunel University, localizada na Inglaterra, HEC Montreal e University of Montreal, 
ambas no Canadá, e University of North Carolina e University System of Georgia, situadas nos Estados Unidos.

O procedimento de acoplamento bibliográfico realizado resultou em cem documentos que continham no mínimo uma citação, a partir dos quais foram criados 5 clusters. As temáticas apresentadas pelos clusters, predominantemente, abordam os efeitos institucionais, intenção de adoção de SI verde, bem como adoção de SI em organizações públicas e privadas, pressões institucionais e dinâmicas de institucionalização de SI.

Destaca-se que em sua maioria os artigos utilizam análises em âmbito organizacional, deixando de explorar as interações do campo, que, por exemplo, engloba o papel da legitimidade na sobrevivência organizacional (CAVALLUZZO; ITTNER, 2004). Ainda, os efeitos institucionais poderiam ser mais explorados, utilizando como objeto de análise a indústria, que oferece um espaço contextual para construir uma nova teoria de SI (CHIASSON; DAVIDSON, 2005), bem como abordar a questão da agência imbricada pelo empreendedorismo institucional, proporcionando uma compreensão de como os indivíduos favorecem as mudanças institucionais mais desejáveis a estes (BATTILANA; LECA; BOXENBAUM, 2009).

Assim, entende-se que a Teoria Institucional ainda é adotada de forma restrita pela comunidade de $\mathrm{SI}$, o que sugere que uma abordagem mais frutífera nos níveis e métodos possa ser desenvolvida (CURRIE, 2009). Tal conclusão culmina com afirmação de Wooten e Hoffman (2008) e Tolbert e Zucker (1999), de que o caminho para a institucionalização da Teoria Institucional é longo, e muitas são as variáveis que ainda podem ser explicadas em busca de respostas a perguntas intermináveis que essa Teoria pode formular.

Com relação à perspectiva metodológica das 20 publicações com o maior número de citações, na análise quanto à natureza e ao tipo de pesquisa das publicações destaca-se que $60 \%$ do conjunto era de natureza qualitativa e $65 \%$ eram do tipo descritivo. Sobressaem-se também nos dados apresentados que $10 \%$ dos estudos são interpretativistas, o que vai de encontro à origem dos estudos da área de $\mathrm{SI}$, que é positivista-funcionalista. Estudos anteriores já haviam apontado para essa possibilidade de fuga de estereótipos metodológicos na área de SI (DANTAS; CALLADO, 2019; RODRIGUES FILHO; LUDMER, 2005). Ainda, quanto ao delineamento, 30\% das pesquisas foram realizadas por meio de estudos de caso e $30 \%$ por meio de pesquisas survey.

Diante dos achados apresentados, este trabalho revela implicações mais amplas no que se refere à utilização da Teoria Institucional como perspectiva de análise, uma vez que, ainda que não tenha fronteiras bem delimitadas e fundamente-se em análises complexas, apresenta inúmeras aplicabilidades nos estudos na área de Sistemas de Informação.

Os fenômenos em SI abordados envolvem principalmente a inovação e a adoção de uma ampla gama de tecnologias, a partir da investigação principalmente de pressões institucionais. A investigação de pressões coercitivas, normativas e miméticas aparenta ser a mais consolidada nos estudos de SI. Tal constatação decorre dos próprios conceitos da Teoria Institucional, uma vez que os mecanismos das pressões institucionais moldam as organizações, o que exige que os pesquisadores se concentrem nos pilares reguladores, normativos e culturais-cognitivos (BUTLER, 2011). Nesse sentido, destaca-se a análise de tais pressões tanto na instituição de SI quanto nas respostas organizacionais às pressões do campo.

No que respeita aos níveis de análise adotados, os estudos da Teoria Institucional desenvolvem-se em três camadas: campo social, campo organizacional e organização. Na presente 
pesquisa evidenciou-se uma concentração no nível organizacional, com os campos organizacionais e o campo social sendo pouco considerados. Tal constatação indica caminhos de pesquisa que carecem de publicações e devem ser mais explorados pelos pesquisadores da área de SI, principalmente devido à padronização de processos e sistemas. Além disso, conforme apontam Mignerat e Rivard (2009), há espaço para contribuições em níveis intermediários de análise, como setores, grupos e processos, que permanecem inexplorados atualmente.

Ademais, evidenciam-se como promissoras pesquisas envolvendo a legitimidade. Tal conceito da Teoria Institucional está relacionado às leis sociais, normas e valores e à garantia de sobrevivência das organizações (MEYER; ROWAN, 1977), e apresenta pouca ênfase nos estudos analisados, podendo ser amplamente relacionado com investigações envolvendo facilitadores e barreiras na adoção e efetivação de SI.

Por fim, sugere-se que pesquisas futuras envolvendo a utilização da Teoria Institucional na área de Sistemas de Informação sejam desenvolvidas de forma a complementar a presente análise, incluindo-se outras bases de dados além da WoS e o aprofundamento em um número maior de artigos, por meio de uma revisão sistemática. Essas sugestões podem suprir as limitações deste estudo, que se concentrou em analisar os artigos indexados na base da WoS, focando nas características gerais e de rede social temática de 128 publicações, porém aprofundando-se no panorama metodológico de apenas 20 publicações com maior número de citações.

\section{REFERÊNCIAS}

AHMADI, H. et al. Hospital Information System adoption: Expert perspectives on an adoption framework for Malaysian public hospitals. Computers in Human Behavior, v. 67, p. 161-189, 2017. Disponível em: https://www.sciencedirect.com/science/article/pii/S0747563216307245. Acesso em: 22 set. 2019.

ARAÚJO, C. A. Bibliometria: evolução histórica e questões atuais. Em Questão, v. 12, n. 1, p. 11-32, 2006. Disponível em: https://www.redalyc.org/pdf/4656/465645954002.pdf. Acesso em: 13 jan. 2020.

BASKERVILLE, R. L.; MYERS, M. D. Fashion waves in information systems research and practice. Mis Quarterly, p. 647-662, 2009. Disponível em: https://www.jstor.org/stable/20650319. Acesso em: 12 out. 2019. BATTILANA, J. Agency and institutions: The enabling role of individuals' social position. Organization, v. 13, n. 5, p. 653-676, 2006. Disponível em: https://doi.org/10.1177/1350508406067008. Acesso em: 11 mar. 2021.

BATTILANA, J.; LECA, B.; BOXENBAUM, E. How actors change institutions: towards a theory of institutional entrepreneurship. Academy of Management annals, v. 3, n. 1, p. 65-107, 2009. Disponível em: https:// doi.org/10.1080/19416520903053598. Acesso em: 22 nov. 2019.

BERENTE, N.; YOO, Y. Institutional contradictions and loose coupling: Postimplementation of NASAs enterprise information system. Information Systems Research, v. 23, n. 2, p. 376-396, 2012. Disponível em: https://pubsonline.informs.org/doi/abs/10.1287/isre.1110.0373. Acesso em: 15 set. 2019.

BHAKOO, V.; CHOI, T. The iron cage exposed: Institutional pressures and heterogeneity across the healthcare supply chain. Journal of Operations Management, v. 31, n. 6, p. 432-449, 2013. Disponível em: https://www.sciencedirect.com/science/article/pii/S0272696313000661. Acesso em: 16 set. 2019.

BRYANT, A. et al. Information Systems history: What IS history? What is IS history? What IS history?... and why even bother with history? 2013. Disponível em: https://link.springer.com/article/10.1057/jit.2013.3. Acesso em: 27 dez. 2019.

BUTLER, T. Compliance with institutional imperatives on environmental sustainability: Building theory on the role of Green is. The Journal of Strategic Information Systems, v. 20, n. 1, p. 6-26, 2011. Disponível em: https://www.sciencedirect.com/science/article/pii/S0963868710000491. Acesso em: 12 set. 2019.

BUTLER, T. An institutional perspective on developing and implementing intranet-and internet-based information systems. Information Systems Journal, v. 13, n. 3, p. 209-231, 2003. Disponível em: https:// onlinelibrary.wiley.com/doi/abs/10.1046/j.1365-2575.2003.00151.x. Acesso em: 12 set. 2019. 
CAVALLUZZO, K. S.; ITTNER, C. D. Implementing performance measurement innovations: evidence from government. Accounting, Organizations and Society, v. 29, n. 3-4, p. 243-267, 2004. Disponível em: https://www.sciencedirect.com/science/article/pii/S0361368203000138. Acesso em: 12 set. 2019.

CHIASSON, M. W.; DAVIDSON, E. Taking industry seriously in information systems research. Mis Quarterly, p. 591-605, 2005. Disponível em: https://www.jstor.org/stable/25148701. Acesso em: 11 set. 2019.

CURRIE, W. Contextualising the IT artefact: towards a wider research agenda for IS using institutional theory. Information Technology \& People, v. 22, n. 1, p. 63-77, 2009. Disponível em: https://www.emeraldinsight.com/doi/abs/10.1108/09593840910937508. Acesso em: 12 set. 2019.

DAMSGAARD, J.; LYYTINEN, K. Contours of diffusion of electronic data interchange in Finland: Overcoming technological barriers and collaborating to make it happen. The Journal of Strategic Information Systems, v. 7, n. 4, p. 275-297, 1998. Disponível em: https://www.sciencedirect.com/science/article/pii/ S0963868798000328. Acesso em: 12 set. 2019.

DANTAS. G. F. M; CALLADO, A. L. C. Quem somos em SI? A história do campo de Sistemas de Informação no Brasil. ENCONTRO NACIONAL DA ASSOCIAÇÃO NACIONAL DE PÓS-GRADUAÇÃO E PESQUISA EM ADMINISTRAÇÃO - ENANPAD, 42., 2018, Curitiba. Anais [...] Curitiba, PR: Anpad, 2018.

DE MELO PEREIRA, F. A. A evolução da teoria institucional nos estudos organizacionais: um campo de pesquisa a ser explorado. Revista Organizações em Contexto, v. 8, n. 16, p. 275-295, 2012. Disponível em: https://www.redalyc.org/pdf/5342/534256501012.pdf. Acesso em: 13 jan. 2020.

DIMAGGIO, P. Interest and agency in institutional theory. Institutional patterns and organizations culture and environment, p. 3-21. Massachusetts: Ballinger Publishing, 1988.

DIMAGGIO, P. J.; POWELL, W. W. A gaiola de ferro revisitada: isomorfismo institucional e racionalidade coletiva nos campos organizacionais. RAE-Revista de Administração de Empresas, v. 45, n. 2, p. 74-89, 2005. Disponível em: http://bibliotecadigital.fgv.br/ojs/index.php/rae/article/view/37123. Acesso em: 11 mar. 2021.

DINIZ, E. H. et al. Inserção Internacional do Campo de Administração da Informação (ADI): análise da formação, publicação e participação em redes de pesquisa. ENCONTRO NACIONAL DA ASSOCIAÇÃO NACIONAL DE PÓS-GRADUAÇÃO E PESQUISA EM ADMINISTRAÇÃO - ENANPAD, 40., 2016. Rio de Janeiro. Anais [...]. Costa do Sauípe: Anpad, 2016.

DONTHU, N.; KUMAR, S.; PATTNAIK, D. Forty-five years of journal of business research: a bibliometric analysis. Journal of Business Research, v. 109, p. 1-14, 2020. Disponível em: https://doi.org/10.1016/j. jbusres.2019.10.039. Acesso em: 11 mar. 2021.

GHOLAMI, R. et al. Senior managers' perception on green information systems (IS) adoption and environmental performance: Results from a field survey. Information \& Management, v. 50, n. 7, p. 431-438, 2013. Disponível em: https://www.sciencedirect.com/science/article/pii/S0378720613000724. Acesso em: 10 set. 2019.

GRÁCIO, M. C. C. Acoplamento bibliográfico e análise de cocitação: revisão teórico-conceitual. Encontros Bibli: revista eletrônica de biblioteconomia e ciência da informação, v. 21, n. 47, p. 82-99, 2016. Disponível em: https://www.redalyc.org/pdf/147/14746959008.pdf. Acesso em: 20 dez. 2019.

GREENWOOD, R. et al. Institutional complexity and organizational responses. Academy of Management Annals, v. 5, n. 1, p. 317-371, 2011. Disponível em: https://journals.aom.org/doi/abs/10.5465/19416520. 2011.590299. Acesso em: 11 mar. 2021.

HEIKKILÄ, J.-P. An institutional theory perspective on e-HRM's strategic potential in MNC subsidiaries. The Journal of Strategic Information Systems, v. 22, n. 3, p. 238-251, 2013. Disponível em: https://www.sciencedirect.com/science/article/pii/S0963868713000516. Acesso em: 20 nov. 2019.

HU, Q.; HART, P.; COOKE, D. The role of external and internal influences on information systems security-a neo-institutional perspective. The Journal of Strategic Information Systems, v. 16, n. 2, p. 153-172, 2007. Disponível em: https://www.sciencedirect.com/science/article/pii/S0963868707000212. Acesso em: 14 set. 2019.

JARNEVING, B. Bibliographic coupling and its application to research-front and other core documents. Journal of Informetrics, v. 1, n. 4, p. 287-307, 2007. Disponível em: https://doi.org/10.1016/j. joi.2007.07.004. Acesso em: 11 mar. 2021.

JENSEN, T. Blegind; KJAERGAARD, A.; SVEJVIG, P. Using institutional theory with sensemaking theory: a case study of information system implementation in healthcare. Journal of Information Technology, v. 24, n. 4, p. 343-353, 2009. Disponível em: https://journals.sagepub.com/doi/abs/10.1057/jit.2009.11. Acesso em: 13 set. 2019. 
JONGSAGUAN, S.; GHONEIM, A. Green IT/IS investments evaluation within the aviation industry. Journal of Enterprise Information Management, 2017. Disponível em: https://www.emerald.com/insight/content/doi/10.1108/JEIM-10-2015-0096/full/html. Acesso em: 13 set. 2019.

KOTZE, C.; VAN BELLE, J.-P.; MCGIBBON, C. Key Drivers of Green Information Systems in South African Listed Companies. In: 2014 5th INTERNATIONAL CONFERENCE-CONFLUENCE THE NEXT GENERATION INFORMATION TECHNOLOGY SUMMIT (CONFLUENCE), 5., 2014. IEEE, 2014. p. 935-940. Disponível em: https:// ieeexplore.ieee.org/abstract/document/6949243/. Acesso em: 11 set. 2019.

KSHETRI, N.; PALVIA, P.; DAI, H. Chinese institutions and standardization: The case of government support to domestic third generation cellular standard. Telecommunications Policy, v. 35, n. 5, p. 399-412, 2011. Disponível em: https://www.sciencedirect.com/science/article/pii/S0308596111000346. Acesso em: 11 set. 2019.

LOPES, I. L. Estratégia de busca na recuperação da informação: revisão da literatura. Ciência da Informação, v. 31, n. 2, 2002. Disponível em: http://www.scielo.br/pdf/\%0D/ci/v31n2/12909.pdf. Acesso em: 13 jan. 2020.

MARTINSONS, M. G. Relationship - based e-commerce: theory and evidence from China. Information Systems Journal, v. 18, n. 4, p. 331-356, 2008. Disponível em: https://onlinelibrary.wiley.com/doi/ abs/10.1111/j.1365-2575.2008.00302.x. Acesso em: 11 set. 2019.

MEYER, J. W.; ROWAN, B. Institutionalized organizations: Formal structure as myth and ceremony. American journal of sociology, v. 83, n. 2, p. 340-363, 1977. Disponivel em: https://www.journals.uchicago.edu/ doi/abs/10.1086/226550. Acesso em: 20 dez. 2019.

MIGNERAT, M.; RIVARD, S. Positioning the institutional perspective in information systems research. Journal of Information Technology, v. 24, n. 4, p. 369-391, 2009. Disponível em: https://journals.sagepub.com/ doi/abs/10.1057/jit.2009.13. Acesso em: 20 dez. 2019.

MIRANDA, S. M.; KIM, Y.-M. Professional versus political contexts: institutional mitigation and the transaction cost heuristic in information systems outsourcing. Mis Quarterly, p. 725-753, 2006. Disponível em: https://www.jstor.org/stable/25148747. Acesso em: 12 set. 2019.

MOTKE, F. D.; DA SILVA RAVANELLO, F.; RODRIGUES, G. O. Teoria institucional: um estudo bibliométrico da última década na Web of Science. Contextus - Revista Contemporânea de Economia e Gestão, v. 14, n. 2, p. 63-86, 2016. Disponível em: http://www.periodicos.ufc.br/contextus/article/view/32266. Acesso em: 13 jan. 2020.

NIELSEN, J. A.; MATHIASSEN, L.; NEWELL, S. Theorization and translation in information technology institutionalization: Evidence from Danish home care. Mis Quarterly, v. 38, n. 1, p. 165-186, 2014. Disponível em: https://www.jstor.org/stable/26554873. Acesso em: 24 out. 2019.

NILASHI, M. et al. Determining the importance of hospital information system adoption factors using fuzzy analytic network process (ANP). Technological Forecasting and Social Change, v. 111, p. 244-264, 2016. Disponível em: https://www.sciencedirect.com/science/article/pii/S0040162516301524. Acesso em: 15 set. 2019.

RAMILLER, N. C.; SWANSON, E. Burton. Organizing visions for information technology and the information systems executive response. Journal of Management Information Systems, v. 20, n. 1, p. 13-50, 2003. Disponível em: https://www.tandfonline.com/doi/abs/10.1080/07421222.2003.11045760. Acesso em: 12 set. 2019.

ROBEY, D.; BOUDREAU, M.-C. Accounting for the contradictory organizational consequences of information technology: Theoretical directions and methodological implications. Information Systems Research, $\mathrm{v}$. 10, n. 2, p. 167-185, 1999. Disponível em: https://pubsonline.informs.org/doi/abs/10.1287/isre.10.2.167. Acesso em: 15 jan. 2020.

RODRIGUES FILHO, J.; LUDMER, G. Sistema de Informação: que ciência é essa? JISTEM: Journal of Information Systems and Technology Management, v. 2, n. 2, p. 151-166, 2005. Disponível em: https://www. redalyc.org/pdf/2032/203219587004.pdf. Acesso em: 26 dez. 2019.

SASSETTI, S. et al. Entrepreneurial cognition and socially situated approach: a systematic and bibliometric analysis. Scientometrics, v. 116, n. 3, p. 1675-1718, 2018. Disponível em: https://link.springer.com/article/10.1007/s11192-018-2809-4. Acesso em: $20 \mathrm{dez} .2019$.

SCOTT, W. R. Approaching adulthood: the maturing of institutional theory. Theory and Society, v. 37, n. 5, p. 427, 2008. Disponível em: https://link.springer.com/article/10.1007/s11186-008-9067-z. Acesso em: $20 \mathrm{dez} .2019$. 
SIA, S. K.; SOH, C. An assessment of package - organisation misalignment: institutional and ontological structures. European Journal of Information Systems, v. 16, n. 5, p. 568-583, 2007. Disponível em: https:// www.tandfonline.com/doi/abs/10.1057/palgrave.ejis.3000700. Acesso em: 24 out. 2019.

SINGLETON, R. A. et al. Approaches to Social Research. New York: Oxford University Press, 1999.

SON, J.-Y.; BENBASAT, I. Organizational buyers' adoption and use of B2B electronic marketplaces: efficiency-and legitimacy-oriented perspectives. Journal of management information systems, v. 24, n. 1, p. 5599, 2007. Disponível em: https://www.tandfonline.com/doi/abs/10.2753/MIS0742-1222240102. Acesso em: 10 set. 2019.

STANDING, C.; SIMS, I.; LOVE, P. IT non-conformity in institutional environments: E-marketplace adoption in the government sector. Information \& Management, v. 46, n. 2, p. 138-149, 2009. Disponível em: https://www.sciencedirect.com/science/article/pii/S0378720609000068. Acesso em: 10 set. 2019.

TOLBERT, P. S.; ZUCKER, L. G. The institutionalization of institutional theory. In: CLEGG, S. R.; HARDY, C. Studying Organization. Theory \& Method. London, Thousand Oaks, New Delhi, 1999. p. 169-184. Cap. 6.

VAN ECK, N. J. et al. A comparison of two techniques for bibliometric mapping: Multidimensional scaling and VOS. Journal of the American Society for Information Science and Technology, v. 61, n. 12, p. 2.4052.416, 2010. Disponível em: https://onlinelibrary.wiley.com/doi/abs/10.1002/asi.21421. Acesso em: 13 jan. 2020.

WEERAKKODY, V.; DWIVEDI, Y. K.; IRANI, Z. The diffusion and use of institutional theory: a cross-disciplinary longitudinal literature survey. Journal of information technology, v. 24, n. 4, p. 354-368, 2009. Disponível em: https://journals.sagepub.com/doi/abs/10.1057/jit.2009.16. Acesso em: 12 out. 2019.

WEB OF SCIENCE, 2020. Home. Disponível em: https://www.webofknowledge.com. Acesso em: 13 jan. 2020.

WOOTEN, M.; HOFFMAN, A. J. Organizational fields: Past, present and future. The Sage handbook of organizational institutionalism, v. 1, p. 131-147, 2008.

YOON, T. E.; GEORGE, J. F. Why aren't organizations adopting virtual worlds? Computers in Human Behavior, v. 29, n. 3, p. 772-790, 2013. Disponível em: https://www.sciencedirect.com/science/article/pii/ S0747563212003329. Acesso em: 10 set. 2019.

ZHANG, P. The IS History Initiative: Looking Forward by Looking Back. Cais, v. 36, p. 24, 2015. Disponível em: https://pdfs.semanticscholar.org/fa00/acf64cd9e1cfdd210d1805558ea078d62b87.pdf. Acesso em: 27 dez. 2019.

ZHENG, D. et al. E-government adoption in public administration organizations: integrating institutional theory perspective and resource-based view. European Journal of Information Systems, v. 22, n. 2, p. 221-234, 2013. Disponível em: https://www.tandfonline.com/doi/abs/10.1057/ejis.2012.28. Acesso em: 17 out. 2019. 